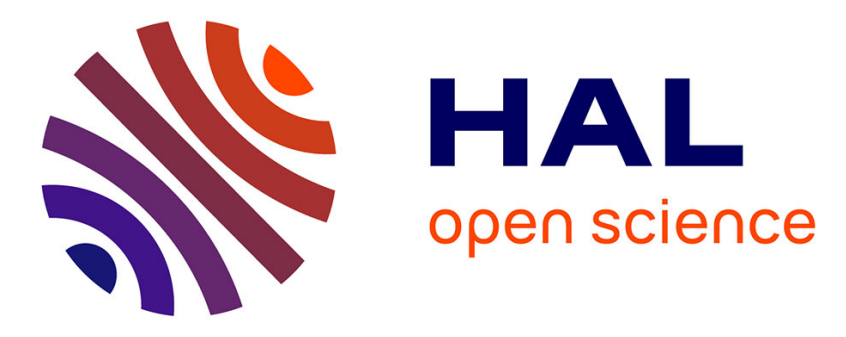

\title{
Preliminary Study of the Perception of Emotions Expressed by Virtual Agents in the context of Parkinson's disease
}

Claire Dussard, Anahita Basirat, Nacim Betrouni, Caroline Moreau, David Devos, François Cabestaing, José Rouillard

\section{To cite this version:}

Claire Dussard, Anahita Basirat, Nacim Betrouni, Caroline Moreau, David Devos, et al.. Preliminary Study of the Perception of Emotions Expressed by Virtual Agents in the context of Parkinson's disease. Workshop on Social Affective Multimodal Interaction for Health (SAMIH) - ICMI 2020, Oct 2020, Utrecht, the Netherlands (virtual), Netherlands. 10.1145/3395035.3425219 . hal-02985246

\section{HAL Id: hal-02985246 https://hal.science/hal-02985246}

Submitted on 2 Nov 2020

HAL is a multi-disciplinary open access archive for the deposit and dissemination of scientific research documents, whether they are published or not. The documents may come from teaching and research institutions in France or abroad, or from public or private research centers.
L'archive ouverte pluridisciplinaire HAL, est destinée au dépôt et à la diffusion de documents scientifiques de niveau recherche, publiés ou non, émanant des établissements d'enseignement et de recherche français ou étrangers, des laboratoires publics ou privés. 


\section{Preliminary Study of the Perception of Emotions Expressed by Virtual Agents in the context of Parkinson's disease}

\author{
Claire Dussard \\ Univ. Lille, CNRS, Centrale Lille, UMR \\ 9189 - CRIStAL - Centre de Recherche \\ en Informatique Signal et \\ Automatique de Lille \\ cdussard@ensc.fr \\ Caroline Moreau \\ Department of Neurology, University \\ Hospital \\ Univ. Lille, Inserm, CHU Lille, U1172 - \\ LilNCog - Lille Neuroscience \& \\ Cognition \\ caroline.moreau@chru-lille.fr
}

\author{
Anahita Basirat \\ Univ. Lille, CNRS, UMR 9193 - \\ SCALab - Sciences Cognitives et \\ Sciences Affectives \\ anahita.basirat@univ-lille.fr
}

\author{
David Devos \\ Departments of Medical \\ Pharmacology \& Neurology, \\ University Hospital \\ Univ. Lille, Inserm, CHU Lille, U1172 - \\ LilNCog - Lille Neuroscience \& \\ Cognition \\ david.devos@chru-lille.fr \\ José Rouillard \\ Univ. Lille, CNRS, Centrale Lille, UMR \\ 9189 - CRIStAL - Centre de Recherche \\ en Informatique Signal et \\ Automatique de Lille \\ jose.rouillard@univ-lille.fr
}

\author{
Nacim Betrouni \\ Univ. Lille, Inserm, CHU Lille, U1172 - \\ LilNCog - Lille Neuroscience \& \\ Cognition \\ nacim.betrouni@inserm.fr
}

\author{
François Cabestaing \\ Univ. Lille, CNRS, Centrale Lille, UMR \\ 9189 - CRIStAL - Centre de Recherche \\ en Informatique Signal et \\ Automatique de Lille \\ francois.cabestaing@univ-lille.fr
}

\begin{abstract}
In the context of Parkinson's disease, this preliminary work aims to study the recognition profiles of emotional faces, dynamically expressed by virtual agents in a Healthy Control (HC) population. In this online experiment, users had to watch 56 trials of two-second animations, showing an emotion progressively expressed by an avatar and then indicate the recognized emotion by clicking a button. 211 participants completed this experiment online as HC. Of the demographics variables, only age influenced negatively recognition accuracy in HC. The intensity of the expression influenced accuracy as well. Interaction effects between gender, emotion, intensity, and avatar gender are also discussed. The results of four patients with Parkinson's Disease are presented as well. Patients tended to have lower recognition accuracy than age-matched HC (59\% for age-matched HC; $45.1 \%$ for patients). Joy, sadness and fear seemed less recognized by patients.
\end{abstract}

\section{CCS CONCEPTS}

- Human-centered computing $\rightarrow$ Empirical studies in $\mathrm{HCI}$; Applied computing $\rightarrow$ Health informatics.

ICMI '20 Companion, October 25-29, 2020, Virtual event, Netherlands

(c) 2020 Association for Computing Machinery.

This is the author's version of the work. It is posted here for your personal use Not for redistribution. The definitive Version of Record was published in Companion Publication of the 2020 International Conference on Multimodal Interaction (ICMI' 20 Companion), October 25-29, 2020, Virtual event, Netherlands, https://doi.org/10.1145/ 3395035.3425219 .

\section{KEYWORDS}

Emotion Recognition, Virtual Agents, Non-verbal communication, Parkinson's Disease

\section{ACM Reference Format:}

Claire Dussard, Anahita Basirat, Nacim Betrouni, Caroline Moreau, David Devos, François Cabestaing, and José Rouillard. 2020. Preliminary Study of the Perception of Emotions Expressed by Virtual Agents in the context of Parkinson's disease. In Companion Publication of the 2020 International Conference on Multimodal Interaction (ICMI '20 Companion), October 2529, 2020, Virtual event, Netherlands. ACM, New York, NY, USA, 5 pages. https://doi.org/10.1145/3395035.3425219

\section{PARKINSON'S DISEASE AND EMOTIONS}

Parkinson's disease (PD) is generally characterized as a motor disorder. However, PD patients are also affected by cognitive and emotional impairments from prodromal to late stages of the disease progression. Some emotional impairments can stem from motor symptoms, such as hypomimia (MDS-UPDRS-III-19 sub score). Also known as "poker face", hypomimia is characterized by a reduction in emotional facial expressions and spontaneous facial movement. Cognition and emotion are different but tightly connected to different degree to trigger appearance of several non-motor symptoms including apathy, impulse compulsive disorders, and mood disorders such as anxiety, dysthymia and depression. Apathy, depression and anxiety have been linked with altered emotion recognition patterns and should be controlled for (Apathy: [19]; depression and anxiety: [7]). Dopamine agonists used as medication for patients 
can also alter their social behavior, notably by triggering impulse compulsive disorders [22].

Deficits in identifying emotion through the facial expressions of others, as well as their own subjective feeling of emotion (alexithymia) are often reported [3]. According to the simulation theory, recognizing emotions in others requires mimicking the expression. When the expression is imitated, the sensory feedback allows for the emotion to be identified. Correlations between difficulties expressing an emotion and recognizing it in others were reported in PD patients [18]. Facial Emotion Recognition (FER) impairment and alexithymia in PD patients might stem from hypomimia.

These impairments can severely impact the social life of the patients. Novice practitioners can be influenced by the masking of their patients, wrongly judging them as less extraverted and more neurotic [21]. Masking is correlated with social rejection and worse partner interaction [17].

We believe that multimodal technology and virtual agents can be used in social skills training for social-affective interactions. Our end goal is the reeducation of the emotional expression of PD patients, improving the amplitude of the emotional facial motricity. To do so, we aim to use virtual agents to represent the facial expression objectives and give online feedback on the facial expressions produced by PD patients. Virtual agents have the advantage of modularity and reproducibility over filmed actors. An infinite combination of parameter settings such as age, gender, race of the avatar, emotion and emotion intensity and duration can be controlled precisely via scripting, instead of requiring actors. Other modalities, such as vocal emotion recognition training could be easily added to the script. However, using avatars requires a construct validity verification. Are they recognized as well as natural expressions? Do avatars worsen the FER deficit in PD patients? Is age or video-game use correlated with recognition rate? If the emotions expressed by those avatars weren't recognized, it could bias the reeducation phase later. Thus, this preliminary study aimed at qualifying the perception of emotions expressed with two avatars before conducting a large comparative study between HC and PD groups.

\section{VIRTUAL AGENTS AND EMOTIONS}

There is extensive research about the recognition rates of emotions expressed by avatars. However, the methodology varies greatly between studies (animation duration, population targeted and construction of the facial expressions) and the technology is evolving fast, making it very difficult to compare results between studies. Most studies featured static, rather than dynamic, material. The effect of dynamic material on the recognition rates seems to depend on age. Dyck et al [9] reported that age moderated the recognition accuracy of pictures of natural and virtual faces (i.e. static material). Beyond the age of 40 , precision decreased for virtual but not natural faces, suggesting media exposure may influence recognition. Beer et al. [5] reported that age-related differences in emotion recognition transcended human faces to virtual agent faces. De Borst et al. [6] concluded that emotion recognition depended on how well the emotions are expressed more than whether they are expressed by a human face. They reported that perception of emotional expression in human-like characters can be processed similarly to human stimuli. However, Kegel et al. [15] showed differences in the perception of fearful dynamic avatars and dynamic human faces. Gender [23], culture [8] and race [11] have been reported to influence FER.

Few studies have been carried out to see if Parkinson's disease emotion recognition deficit translates with dynamic virtual characters. Argaud et al. [2] has shown that PD patients had significantly lower accuracy scores compared to controls for joy and neutral expressions but not anger. The dynamic nature of the material matters. Presenting PD patients with static faces could worsen their recognition accuracy compared to dynamic faces, artificially inflating their FER deficit [14]. Ho et al. [12] has recently shown deficits in anger and disgust recognition with dynamic human faces. They reported that age, slow mentation and poor cognition were associated with FER deficit in PD patients, and not in age-matched controls. It could be hypothesized that the recognition deficits of Parkinson's disease patients will subsist for dynamic virtual agents. This study aims to qualify how facial expressions and emotions expressed by virtual agents are perceived by people. PD patients have been suggested to trust avatar faces more than human faces [13].

As we aim to target daily social interactions, we chose to investigate dynamic material, to maximize the ecological aspect of the manipulation. Our hypothesis is that using avatars could be an effective tool for the reeducation of emotional facial expression perception and production for patients.

\section{ONLINE EXPERIMENTATION AND PRELIMINARY RESULTS}

\subsection{Methods}

We chose to study the six basic emotions: joy, sadness, fear, anger, disgust and surprise [10], allowing our results to be compared to the literature. The contempt facial expression was added as a control. In this 5-minute-long experiment, we presented the subjects with an avatar progressively expressing an emotional facial expression (EFE) for 2 seconds (as in $[23,14]$ ). After the end of the animation, the avatar disappears and an interface consisting of 8 buttons (the order was not randomized) appears. The subjects could select one of the six basic emotions and had the option to select "Other" or "I don't know". Subjects were instructed to be as precise as possible.

The experiment featured 56 trials, 28 with a Caucasian female avatar and 28 with a Caucasian male avatar. The presentation order of the emotions was pseudo-randomized: each combination of the parameters was presented twice to the subject. Two thresholds representing $50 \%$ and $100 \%$ of the maximum intensity were introduced (see Figure 1). It should be noted that those thresholds are mathematical constructs, based on the blendshape model of facial animation (see [1]). A half-threshold expression does necessarily mean the real-life emotion is of half intensity.

Each trial featured a combination of 3 parameters: avatar gender, intensity and emotion. In total, the experiment featured 2 repetitions $\times 2$ genders $\times 2$ thresholds $\times 7$ emotions $=56$ trials. Both avatars had the same technology and scripts for expressions. We used the native Genesis 8 avatar implementations of joy, surprise, fear and anger. Sadness, disgust and control were scripted using the blendshapes. Blendshapes represent a linear weighted sum of the target faces, allowing to represent an emotional expression by 

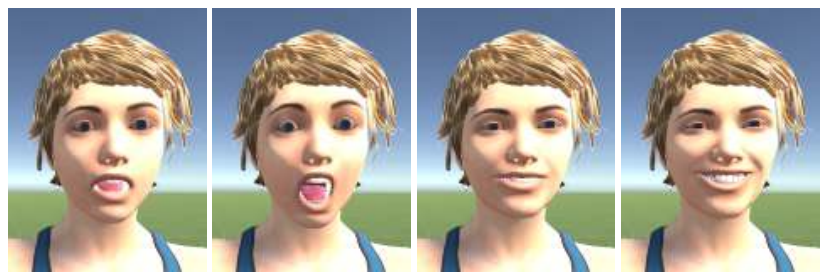

surprise $50 \% \quad$ surprise $100 \%$

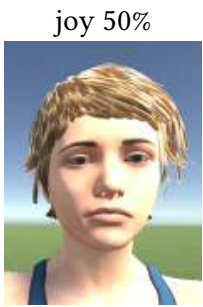

joy $100 \%$
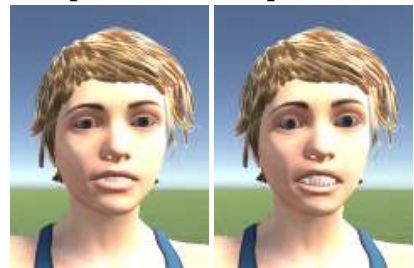

sadness $50 \%$

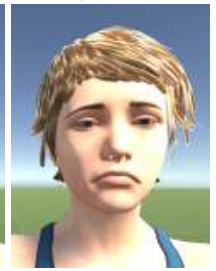

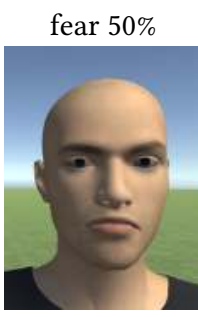

disgust $50 \%$ fear $100 \%$

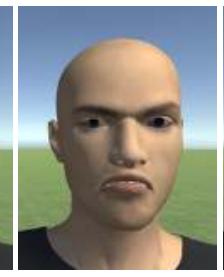

disgust $100 \%$

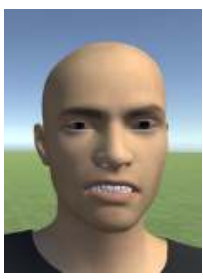

anger $50 \%$
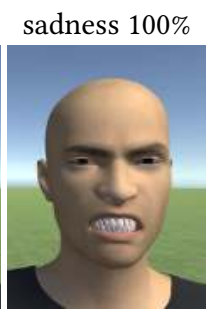

anger $100 \%$
Figure 1: Facial emotion recognition stimulus material with two thresholds.

approximating facial muscle actions [1]. We scripted their incrementation frequency accordingly to keep constant the duration of the animation across thresholds. Gender, age, frequency of use of a computer or cellphone and frequency of videogame playing were collected as well. The software was created with Unity3D. A WebGL version was hosted on a French server from July 10th, 2020 to August 15th, 2020.

\subsection{Participants}

242 French-speaking subjects were recruited as Healthy Controls (HC) through mailing lists. 211 of them finished the study. 100 women and 111 men completed the study. The mean age was 36.6 years old $(\mathrm{SD}=14.6)$. Men participants were older than women (Mann-Whitney-Wilcoxon (MWW) test $\mathrm{W}=4442, \mathrm{p}=0.012 ; 38.1$ for men; 35 for women). Videogame-use was coded as such: 4: every day; 3 : every week; 2 : every month; 1 : less often or never. Mean videogame use was $2.05(\mathrm{SD}=1.16)$. It differed significantly between genders ( 2.32 for men; 1.75 for women; MWW test, $\mathrm{W}=$ $3530, \mathrm{p}=0.0005)$. Four patients with Parkinson's disease (PD) were recruited ( 3 men, 1 woman). The mean age was 61 years old (SD $=11.1$ ), higher than HC controls (Welch's $\mathrm{t}$-test, $\mathrm{t}=4.31, \mathrm{p}=0.02$ ). Mean videogame use was $1.5(\mathrm{SD}=1)$, it did not differ from $\mathrm{HC}$. All participants consented to have their results and demographic data used anonymously in this study.

\subsection{Overall results and demographic variables}

Mean global recognition accuracy (RA, percentage of the trials where the correct emotion was selected) for $\mathrm{HC}$ was $63.5 \%$. Gender and video game use did not moderate mean RA. Only age was a significant moderator of mean RA, with an average reduction effect on mean RA of $-0.2 \%$ per year of age (age 18 to 84; Pearson correlation, $\mathrm{t}=5.03, \mathrm{p}<0.001, \mathrm{r}=-0.33)$. Mean response selection time was $2.3 \mathrm{~s}$ ( $2.4 \mathrm{~s}$ for age-matched $\mathrm{HC})$.

\subsection{Avatar gender effect}

For HC, gender congruence of the avatar did not affect the mean RA. In this experiment, the gender of the avatar was not randomized. After analysis, this effect of order was not significant. A significant interaction effect between threshold and avatar gender was found in $\mathrm{HC}(\mathrm{F}(1,1)=8.90, \mathrm{p}=0.0003$ for HC. It was only significant in half-threshold trials: subtle emotions expressed by the male avatar were better recognized than those of the female avatar (MWW test, $\mathrm{W}=3620700, \mathrm{p}=0.004)$. We found an interaction effect between emotion and avatar gender $(F(1,1)=9.41, p=0.0002)$. Joy and anger were better recognized with the male avatar (as reported for joy in [20]). Sadness was better recognized with the female avatar.

\subsection{Threshold effect}

Emotion threshold influenced RA $(\mathrm{F}(1,1)=139.29, \mathrm{p}<0.0001)$. For $\mathrm{HC}$, maximum-threshold trials had a mean RA of $68.2 \%$ versus $58.9 \%$ for half-intensity trials (Welch's $\mathrm{t}$-test, $\mathrm{t}=10.42, \mathrm{p}<0.001$ ). As shown by Figure 2, there was a significant interaction effect between emotion and threshold $(\mathrm{F}(1,1)=17.97, \mathrm{p}<0.0001$ for $\mathrm{HC})$. For HC, RA increased with the maximum threshold for 3 out of 7 emotions (anger, sadness, fear) and worsened for 2 emotions (disgust, surprise). There was a significant interaction effect between age and threshold $(\mathrm{F}(1,1)=6.30, \mathrm{p}=0.012)$. Maximum threshold increased RA by $12.7 \%$ for participants over 48 years old, and $8.3 \%$ for younger participants (MWW test, $\mathrm{W}=3333900, \mathrm{p}=0.022$ ).

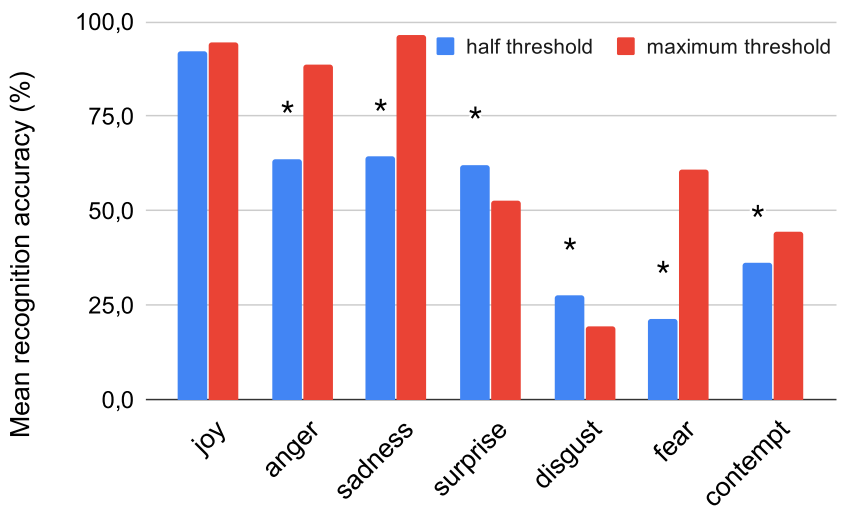

Figure 2: Effect of emotion and threshold on mean recognition accuracy in HC. Chance performance: $14.3 \%$. $\left({ }^{*}\right.$ : $\left.p<0.01\right)$

\subsection{Emotion effect}

Emotion had a significant effect on the recognition accuracy of the trial $(\mathrm{F}(1,1)=2697.5, \mathrm{p}<0.0001)$. Mean RA differed highly between 
emotions. For HC, accurately recognized emotions were joy (95.5\%), sadness $(88.9 \%)$, anger $(79.8 \%)$ and surprise $(70.3 \%)$, as reported in Figure 2. Poorly recognized emotions were fear (48.9\%), contempt the control option which should have been recognized as "Other" (44.3\%) and disgust (16.9\%, just above chance performance). Disgust and fear were the two emotions that were the least recognized overall. Disgust was mistaken for anger (79.7\% of the errors). This error has been reported before $[5,10]$ and disgust is reportedly difficult to convey with avatar faces $[5,16]$. Fear was mistaken for surprise ( $44.4 \%$ of the errors). For HC, contempt was mistaken for joy ( $83.3 \%$ of the errors). We found a significant interaction effect between gender and emotion $(F(1,1)=13.76, p=0.0002)$. Men recognized joy and surprise better than women. Women recognized fear and contempt (other) better than men.

\subsection{Patients' results}

Mean global RA for patients was $45.1 \%$, as compared to $59 \%$ in age-matched HC. Age-matched HC seemed to perform better than patients for joy (replicating [2]), sadness and fear. Patient's fear RA was way below age-matched group's fear RA. Patients seemed to recognize anger surprise, disgust and contempt as well as agematched HC did.

The mean response selection time was 5.8 s for patients. Patients included in this study had good motor condition. Their higher response time could not be attributed to a higher reaction time overall.

As reported in Figure 3, accurately recognized emotions were sadness (65.6\%), joy (62.5\%), surprise (56.3\%) and anger (53.1\%). Poorly recognized emotions were contempt (50\%), disgust (21.9\%) and fear (6.3\%). Disgust was mistaken for anger (48\% of the errors). Fear was mistaken for surprise (26.7\% of the errors). Contempt was mistaken for surprise (37.5\%) and joy (25\%).

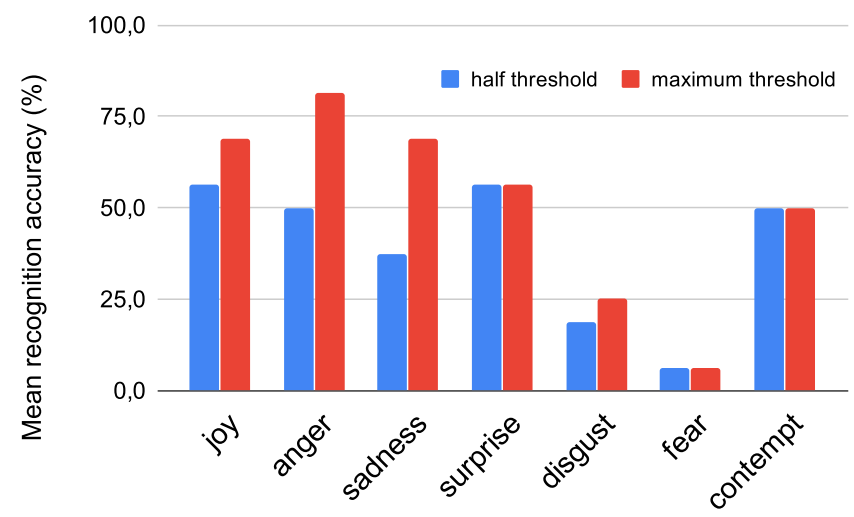

Figure 3: Effect of emotion and threshold on mean recognition accuracy in patients.

Maximum-threshold trials had a mean overall RA of $50.1 \%$ versus $39.3 \%$ for half-intensity trials. RA increased with the maximum threshold for 4 emotions (joy, anger, sadness, disgust) and remained unaffected for the remaining 3 others (contempt, fear, surprise).

\section{CONCLUSION}

In this article, we present the results of an online experiment. Those results validate our approach's feasibility: our Proof of Concept was usable by a reasonably large number of participants, patients and healthy controls.

A few limits of our study should be noted. As stated by Ricciardi et al. [18], the very high RA of the joy expression might stem from a design bias: being the only positive emotion of the 6 described by Ekman might have inflated its recognition rate. Including more positive emotions (pride, euphoria) would allow to mitigate this effect but those emotions are complex constructions, with less prototypical facial expressions. The closed-choice paradigm that was used was also a help to participants but was mitigated by the availability of "other" and "I don't know" options.

As three of the seven emotions tested were constructed, it would be necessary to assess their construct validity. All emotions except fear in the patient's group were recognized above chance threshold. Those avatars could be suitable candidates for future studies as EFE training objectives and feedback. Future experiments could include academically validated avatar expressions, such as those presented by Broekens et al. [25] and Yu et al. [24]. Our recognition accuracy results are comparable to those reported with dynamic avatars in $[2,16]$.

As shown by Aviezer et al. [4], emotion perception is largely dependent on context, including situation, body language and prosody. Studying the recognition patterns of EFE depending on the congruence of the context in PD patients would allow to characterize more precisely the FER deficit. The removal of body language and context from experiments might artificially inflate the FER deficits of patients.

As avatars were used, the recognition rates reported reflect the adequacy between the participant's mental representation of an emotional expression and the avatar's actual expression. Including more context might allow more ecological observations to conclude on the nature of the FER deficit observed in patients. Future experiments will include more patients and could feature EEG, EMG and eye-tracking measures to investigate the nature of the differences in emotion recognition profiles between groups.

\section{REFERENCES}

[1] Ken Anjyo. 2018. Blendshape Facial Animation. Springer International Publishing, Cham, 2145-2155. https://doi.org/10.1007/978-3-319-14418-4_2

[2] Soizic Argaud, Sylvain Delplanque, Jean-François Houvenaghel, Manon Auffret, Joan Duprez, Marc Vérin, Didier Grandjean, and Paul Sauleau. 2016. Does Facial Amimia Impact the Recognition of Facial Emotions? An EMG Study in Parkinson's Disease. PLOS ONE 11, 7 (July 2016), 1-20. https://doi.org/10.1371/ journal.pone.0160329

[3] Soizic Argaud, Marc Vérin, Paul Sauleau, and Didier Grandjean. 2018. Facial emotion recognition in Parkinson's disease: a review and new hypotheses. Movement Disorders 33, 4 (2018), 554-567. https://doi.org/10.1002/mds.27305

[4] Hillel Aviezer, Ran R. Hassin, Jennifer Ryan, Cheryl Grady, Josh Susskind, Adam Anderson, Morris Moscovitch, and Shlomo Bentin. 2008. Angry, Disgusted, or Afraid?: Studies on the Malleability of Emotion Perception. Psychological Science 19, 7 (2008), 724-732. https://doi.org/10.1111/j.1467-9280.2008.02148.x PMID: 18727789.

[5] Jenay M. Beer, Cory-Ann Smarr, Arthur D. Fisk, and Wendy A. Rogers. 2015. Younger and older users' recognition of virtual agent facial expressions. International Journal of Human-Computer Studies 75 (2015), 1-20. https://doi.org/10. 1016/j.ijhcs.2014.11.005

[6] Aline W. de Borst and Beatrice de Gelder. 2015. Is it the real deal? Perception of virtual characters versus humans: an affective cognitive neuroscience perspective. Frontiers in Psychology 6 (2015), 576. https://doi.org/10.3389/fpsyg.2015.00576 
[7] Liliana R. Demenescu, Rudie Kortekaas, Johan A. den Boer, and André Aleman. 2010. Impaired Attribution of Emotion to Facial Expressions in Anxiety and Major Depression. PLOS ONE 5, 12 (Dec. 2010), 1-5. https://doi.org/10.1371/ journal.pone.0015058

[8] Birgit Derntl, Ute Habel, Simon Robinson, Christian Windischberger, Ilse KryspinExner, Ruben C. Gur, and Ewald Moser. 2012. Culture but not gender modulates amygdala activation during explicit emotion recognition. BMC Neuroscience 54, 13 (May 2012), 54-54. https://doi.org/10.1186/1471-2202-13-54

[9] Miriam Dyck, Maren Winbeck, Susanne Leiberg, Yuhan Chen, Rurben C. Gur, and Klaus Mathiak. 2008. Recognition Profile of Emotions in Natural and Virtual Faces. PLOS ONE 3, 11 (Nov. 2008), 1-8. https://doi.org/10.1371/journal.pone.0003628

[10] Paul Ekman, Wallace Friesen, Maureen O'Sullivan, Anthony Chan, Irene Diacoyanni-Tarlatzis, Karl Heider, Rainer Krause, William LeCompte, Tom Pitcairn, and Pio Ricci Bitti. 1987. Universals and Cultural Differences in the Judgments of Facial Expressions of Emotion. Fournal of personality and social psychology 53 (Nov. 1987), 712-7. https://doi.org/10.1037/0022-3514.53.4.712

[11] Amy G. Halberstadt, Alison N. Cooke, Pamela W. Garner, Sherick A. Hughes, Dejah Oertwig, and Shevaun D. Neupert. 2020. Racialized emotion recognition accuracy and anger bias of children's faces. Emotion (2020), 15. https://doi.org/ 10.1037/emo0000756

[12] Mary Wen-Reng Ho, Sarina Hui-Lin Chien, Ming-Kuei Lu, Jui-Cheng Chen, Yu Aoh, Chun-Ming Chen, Hsien-Yuan Lane, and Chon-Haw Tsai. 2020. Impairments in face discrimination and emotion recognition are related to aging and cognitive dysfunctions in Parkinson's disease with dementia. Scientific Reports 10, 1 (March 2020), 4367. https://doi.org/10.1038/s41598-020-61310-w

[13] Andrija Javor, Gerhard Ransmayr, Walter Struhal, and René Riedl. 2016. Parkinson Patients' Initial Trust in Avatars: Theory and Evidence. PLOS ONE 11, 11 (Nov. 2016), 1-21. https://doi.org/10.1371/journal.pone.0165998

[14] Yayoi Kan, Mitsuru Kawamura, Yukihiro Hasegawa, Satoshi Mochizuki, and Katsuki Nakamura. 2002. Recognition Of Emotion From Facial, Prosodic And Written Verbal Stimuli In Parkinson's Disease. Cortex 38, 4 (2002), 623-630. https://doi.org/10.1016/S0010-9452(08)70026-1

[15] Lorena C. Kegel, Peter Brugger, Sascha Frühholz, Thomas Grunwald, Peter Hilfiker, Oona Kohnen, Miriam L. Loertscher, Dieter Mersch, Anton Rey, Teresa Sollfrank, Bettina K. Steiger, Joerg Sternagel, Michel Weber, and Hennric Jokeit. 2020. Dynamic human and avatar facial expressions elicit differential brain responses. Social Cognitive and Affective Neuroscience 15, 3 (March 2020), 303-317. https://doi.org/10.1093/scan/nsaa039

[16] Samuel Marcos-Pablos, Emilio González-Pablos, Carlos Martín-Lorenzo, Luis A Flores, Jaime Gómez-García-Bermejo, and Eduardo Zalama. 2016. Virtual Avatar for Emotion Recognition in Patients with Schizophrenia: a Pilot Study. Frontier in Human Neuroscience 10 (2016), 421. https://doi.org/10.3389/fnhum.2016.00421

[17] Kate Perepezko, Jared T. Hinkle, Melissa D. Shepard, Nicole Fischer, Martinus P. G. Broen, Albert F. G. Leentjens, Joseph J. Gallo, and Gregory M. Pontone. 2019. Social role functioning in Parkinson's disease: a mixed-methods systematic review. International Journal of Geriatric Psychiatry 34, 8 (2019), 1128-1138 https://doi.org/10.1002/gps.5137

[18] Lucia Ricciardi, Federica Visco-Comandini, Roberto Erro, Francesca Morgante, Matteo Bologna, Alfonso Fasano, Diego Ricciardi, Mark J. Edwards, and James Kilner. 2017. Facial Emotion Recognition and Expression in Parkinson's Disease: An Emotional Mirror Mechanism? PLOS ONE 12, 1 (Jan. 2017), 1-16. https: //doi.org/10.1371/journal.pone.0169110

[19] Gabriel H. Robert, Florence Le Jeune, Clement Lozachmeur, Sophie Drapier, Thibaut Dondaine, Julie Péron, Jean-Francois Houvenaghel, David Travers, Paul Sauleau, Bruno Millet, Marc Vérin, and Dominique Drapier. 2014. Preoperative factors of apathy in subthalamic stimulated Parkinson disease. Neurology 83, 18 (2014), 1620-1626. https://doi.org/10.1212/WNL.0000000000000941

[20] John Eric Steephen, Samyak Raj Mehta, and Raju Surampudi Bapi. 2018. Do We Expect Women to Look Happier Than They Are? a Test of Gender-Dependent Perceptual Correction. Perception 47, 2 (2018), 232-235. https://doi.org/10.1177/ 0301006617745240 PMID: 29199878.

[21] Linda Tickle-Degnen and Kathleen Doyle Lyons. 2004. Practitioners' impressions of patients with Parkinson's disease: the social ecology of the expressive mask. Social Science \& Medicine 58, 3 (2004), 603-614. https://doi.org/10.1016/S02779536(03)00213-2

[22] Valerie Voon, Jennifer Gao, Christina Brezing, Mkael Symmonds, Vindhya Ekanayake, Hubert Fernandez, Raymond J. Dolan, and Mark Hallett. 2011. Dopamine agonists and risk: impulse control disorders in Parkinson's disease Brain 134, 5 (May 2011), 1438-1446. https://doi.org/10.1093/brain/awr080

[23] Tanja S. H. Wingenbach, Chris Ashwin, and Mark Brosnan. 2018. Sex differences in facial emotion recognition across varying expression intensity levels from videos. PLOS ONE 13, 1 (Jan. 2018), 1-18. https://doi.org/10.1371/journal.pone. 0190634

[24] Hui Yu, Oliver G. B. Garrod, and Philippe G. Schyns. 2012. Perception-driven facial expression synthesis. Computers \& Graphics 36, 3 (2012), 152-162. https //doi.org/10.1016/j.cag.2011.12.002 Novel Applications of VR.

[25] Juyong Zhang, Keyu Chen, and Jianmin Zheng. 2020. Facial Expression Retargeting from Human to Avatar Made Easy. IEEE transactions on visualization and 